\title{
Analysis of IL2/IL21 Gene Variants in Cholestatic Liver Diseases Reveals an Association with Primary Sclerosing Cholangitis
}

\author{
Johannes Stallhofer ${ }^{a}$ Gerald Ulrich Denk ${ }^{a}$ Jürgen Glas ${ }^{a}$ c \\ Rüdiger Paul Laubender ${ }^{b}$ Burkhard Göke ${ }^{a} \quad$ Christian Rust $^{a}$ \\ Stephan Brand ${ }^{\mathrm{a}} \quad$ Thomas Pusl $^{\mathrm{a}, \mathrm{d}}$ \\ ${ }^{a}$ Department of Medicine II and ${ }^{b}$ Institute of Medical Informatics, Biometry and Epidemiology, University Hospital \\ Munich-Grosshadern, ' Department of Preventive Dentistry and Periodontology, Ludwig Maximilian University, \\ Munich, and ${ }^{\mathrm{d}}$ First Medical Clinic, Klinikum Augsburg, Augsburg, Germany
}

\section{Key Words}

IL2 • IL21 • Polymorphism • Primary biliary cirrhosis •

Primary sclerosing cholangitis • Ulcerative colitis •

Autoimmune disease $\cdot$ Th17 cells

\begin{abstract}
Background/Aims: The chromosome 4q27 region harboring IL2 and IL21 is an established risk locus for ulcerative colitis (UC) and various other autoimmune diseases. Considering the strong coincidence of primary sclerosing cholangitis (PSC) with UC and the increased frequency of other autoimmune disorders in patients with primary biliary cirrhosis (PBC), we investigated whether genetic variation in the IL2/ IL21 region may also modulate the susceptibility to these two rare cholestatic liver diseases. Methods: Four strongly UC-associated single nucleotide polymorphisms (SNPs) within the KIAA1109/TENR/IL2/IL21 linkage disequilibrium block were genotyped in 124 PBC and 41 PSC patients. Control allele frequencies from 1,487 healthy, unrelated Caucasians were available from a previous UC association study. Results: The minor alleles of all four markers were associated with a decreased susceptibility to PSC (rs13151961: $p=0.013$, odds ratio (OR) 0.34; rs13119723: $p=0.023$, OR 0.40; rs6822844: $p=0.031$, OR 0.41; rs6840978: $p=0.043$, OR 0.46).
\end{abstract}

Moreover, a haplotype consisting of the four minor alleles also had a protective effect on PSC susceptibility $(p=0.0084$, OR 0.28). A haplotype of the four major alleles was independently associated with PSC when excluding the patients with concomitant inflammatory bowel disease $(p=0.033$, OR 4.18). Conclusion: The IL2/IL21 region may be one of the highly suggestive but so far rarely identified shared susceptibility loci for PSC and UC.

Copyright $\odot 2011$ S. Karger AG, Basel

\section{Introduction}

Primary biliary cirrhosis (PBC) and primary sclerosing cholangitis (PSC) are chronic cholestatic liver diseases characterized by an immune-mediated destruction of the biliary system, resulting in biliary fibrosis, cirrhosis, and finally chronic liver failure $[1,2]$. PBC is deemed to be a model autoimmune disease due to its striking female predominance and the existence of a disease-specific au-

J.S. and G.U.D. contributed equally to this work. S.B. and T.P. share senior authorship.

\section{KARGER}

Fax +41613061234 E-Mail karger@karger.ch www.karger.com
Stephan Brand, MD

Department of Medicine II, Klinikum Grosshadern, Ludwig Maximilian University DE-81377 Munich (Germany)

Tel. +49 897095 2295, Fax +49 8970955291

E-Mail stephan.brand@med.uni-muenchen.de 
toantibody directed against the E2 subunit of the mitochondrial pyruvate dehydrogenase complex [2]. In contrast, PSC may rather be caused by a dysregulated immune response to bacterial or viral antigens entering the portal circulation due to increased permeability of the colonic mucosa - or by activated T lymphocytes undergoing enterohepatic circulation [1]. Consistent with that, there is a strong association of PSC with inflammatory bowel disease (IBD). In western countries, between 53 and $98 \%$ of PSC patients have concomitant IBD, primarily ulcerative colitis (UC) $[3,4]$. Conversely, the prevalence of PSC among patients with UC ranges from 2 to $7.6 \%[3,4]$

However, while recent genome-wide association studies have revealed several overlapping susceptibility loci (reviewed in [5]) for a variety of immune-mediated diseases including PBC [6], the search for common genetic risk factors for UC and PSC provided results that were well behind expectations. Only 2 of 15 established UC loci tested, namely $\mathrm{G}$ protein-coupled bile acid receptor 1 (GPBAR1; 2q35) and macrophage-stimulating 1 (MST1; 3p21), showed moderate association with PSC in a Scandinavian cohort [7]. Apart from glypican 6 (GPC6; 13q31), which is not implicated in UC, the first genome-wide association study in PSC failed to detect susceptibility loci outside the HLA complex on chromosome 6p21, whose role in PSC susceptibility has already been extensively studied [7].

Given the limited data on shared susceptibility loci for UC and PSC, we aimed to evaluate the role of four genetic polymorphisms in the IL2/IL21 region (rs13151961, rs13119723, rs6822844 and rs6840978) in our cohort of PSC patients on a candidate gene basis. These polymorphisms are strongly associated with UC [8-11], but were not included in the above-mentioned genome-wide analysis [7] and were not part of the 15 UC loci previously analyzed in PSC [7].

The association of the IL2/IL21 region on chromosome 4q27 with celiac disease [12], type 1 diabetes [1315], rheumatoid arthritis [14, 16, 17], psoriatic arthritis [18], juvenile idiopathic arthritis $[19,20]$, systemic lupus erythematosus [17, 21], Sjögren's syndrome [17] and Grave's disease [15] suggests this region as a general autoimmune risk locus and makes it also an interesting candidate locus for PBC. The frequency of classic autoimmune diseases is increased in both PSC patients and PBC patients and their first-degree relatives [22, 23].

In order to examine a potential association with $\mathrm{PBC}$ or PSC, we determined allele frequencies and haplotype frequencies of the four aforementioned IL2/IL21-poly- morphisms in two well-characterized patient cohorts and compared them with a large group of healthy control subjects.

\section{Methods}

Patients and Control Subjects

The study included 124 PBC and 41 PSC patients of European Caucasian descent. All patients were recruited at Grosshadern University Hospital, Munich, Germany. PBC was diagnosed using standard criteria: presence of anti-mitochondrial antibodies (AMA) in serum, elevation of cholestatic liver enzymes and/or disease-specific hepatic histopathology [24]. The diagnosis of PSC was based on biochemical changes consistent with chronic cholestasis in coincidence with characteristic intrahepatic and/or extrahepatic alterations of the bile ducts shown by endoscopic retrograde or magnetic resonance cholangiopancreatography [25]. Detailed clinical data for all patients were obtained by analysis of patient charts.

Genotyping results of 1,487 healthy unrelated European Caucasian controls for the herein tested SNPs in the IL2/IL21 region were available from a previous study investigating their role in UC [10]. The epidemiological characteristics of the three study groups are given in table 1. Written informed consent was obtained from all participants. Ethical approval was given by the Ethics Committee of the Medical Faculty of Ludwig Maximilian University, Munich. The study adhered to the Ethical Principles for Medical Research Involving Human Subjects of the Helsinki Declaration. All data were stored in a coded, anonymized database.

SNP Selection and Genotyping

A roughly $440 \mathrm{~kb}$-long region of pronounced linkage disequilibrium on chromosome 4q27 comprising the four genes KIAA1109, TENR, IL2, and IL21 has initially been identified as the strongest susceptibility locus for celiac disease outside the HLA complex by a genome-wide association scan [12]. The four SNPs rs13151961 $(A>G), \operatorname{rs} 13119723(A>G), \operatorname{rs6822844}(G>T)$, and rs6840978 (C>T) were the strongest disease associated polymorphisms within this gene cluster in the initial celiac disease scan and all showed highly significant association with UC in two independent studies $[8,10]$. Therefore, we chose these four SNPs to determine whether genetic variation in the chromosome $4 \mathrm{q} 27$ region is also implicated in the susceptibility to PBC or PSC.

Venous EDTA blood samples were obtained from all patients and genomic DNA was isolated from peripheral blood leukocytes by using the DNA Blood Mini Kit (Qiagen, Hilden, Germany). Genotyping was performed by melting curve analysis in a LightCycler ${ }^{\circledR} 480$ instrument (Roche Diagnostics, Mannheim, Germany) as described previously [10] and is specified in the online suppl. appendix 1 (www.karger.com/doi/10.1159/000321619). The overall genotyping success rate was $99.0 \%$ for controls, $99.4 \%$ for PBC and $100 \%$ for PSC patients.

\section{Statistical Analysis}

Before statistical analysis, conformance with the Hardy-Weinberg equilibrium was computed for all markers. The genotype frequencies predicted by allele frequencies were compared to the observed genotypes. Therefore, an exact test integrated in Haplo- 
Table 1. Clinical characteristics of the study population

\begin{tabular}{lccc}
\hline & PBC $(\mathrm{n}=124)$ & PSC $(\mathrm{n}=41)$ & Controls $(\mathrm{n}=1,487)$ \\
\hline Male, $\mathrm{n}(\%)$ & $3(2.4)$ & $28(68.3)$ & $928(62.4)$ \\
Female, $\mathrm{n}(\%)$ & $121(97.6)$ & $13(31.7)$ & $559(37.6)$ \\
Concomitant IBD, $\mathrm{n}(\%)$ & $2(1.6)$ & $23(56.1)$ & \\
$\quad$ Ulcerative colitis, $\mathrm{n}(\%)$ & $1(0.8)$ & $21(51.2)$ & $1(2.4)$ \\
$\quad 1(0.8)$ & 0 & $1(2.4)$ & \\
$\quad$ Crohn's disease, $\mathrm{n}(\%)$ & $10(8.1)$ & $3(7.3)$ & \\
Indeterminate colitis, $\mathrm{n}(\%)$ & $63.9 \pm 12.1(19-89)$ & $40.4 \pm 13.5(21-92)$ & $46.0 \pm 10.7(18-71)$ \\
AIH overlap syndrome, $\mathrm{n}(\%)$ & $48.9 \pm 9.6(19-70)$ & $31.7 \pm 14.9(13-86)$ & \\
Mean age \pm SD (range), years & & & \\
Mean age at diagnosis \pm SD (range), years & $48.0 \pm 22.6(32-64)$ & $23.2 \pm 15.3(10-82)$ & \\
Mean age at diagnosis of concomitant & $15(12.1)$ & $4(9.8)$ & $1(2.4)$ \\
$\quad$ IBD \pm SD (range), years & 0 & 0 & \\
Liver transplantation, $\mathrm{n}(\%)$ & $2(1.6)$ & & \\
Cholangiocarcinoma, $\mathrm{n}(\%)$ & & \\
Hepatocellular carcinoma, $\mathrm{n}(\%)$ &
\end{tabular}

view 4.2 [26] was applied to the combined dataset of all three cohorts. Haploview provided $\mathrm{r}^{2}$ linkage disequilibrium (LD) statistics for the four tested polymorphisms and was further used for the evaluation of haplotype counts and frequencies. It analyzes haplotype association by $\chi^{2}$ testing and performs permutations to correct for multiple testing. Since in the PSC cohort, some haplotype counts were 5 or less, we show Fisher's exact test p values in brackets in table 3. For single marker association analysis, case and control allele counts - or genotypes where indicated - were compared with Fisher's exact test. Blaker's exact test [27] was used where denoted by $P_{\mathrm{BL}}$. A two-tailed $\mathrm{p}<0.05$ was considered statistically significant. Odds ratios (ORs) were calculated with a $95 \%$ confidence interval (CI) and statistical power of the odds ratios was defined with SNP_tools [28]. Fisher's exact test was performed with SPSS 15.0 software (SPSS Inc., Chicago, Ill., USA). Blaker's exact test was computed by $\mathrm{R}$ (version 2.11.1) software $(\mathrm{R}$ Development Core Team, Vienna, Austria). Student's t test was applied for quantitative variables and was calculated with Microsoft Excel (Microsoft Corp., Redmond, Wash., USA).

\section{Results}

\section{Patient Characteristics}

The main clinical characteristics of the PBC and PSC patients are shown in table 1 . One hundred and twentyone $(97.6 \%)$ of the $124 \mathrm{PBC}$ patients were female reflecting the known substantial female preponderance of this disease. Ten (8.1\%) of the PBC patients had an autoimmune hepatitis (AIH) overlap syndrome. The mean age at diagnosis of $\mathrm{PBC}$ was $48.9 \pm 9.6$ SD years. Histological staging was available for 64 of the 124 PBC patients. Of these, 23 (35.9\%) showed advanced disease (stage III-IV), whereas 41 (64.1\%) were in stage I or II. Fifteen of the 124
PBC patients (12.1\%) had already undergone liver transplantation. This percentage was similar in the PSC group (4 of 41 patients; 9.8\%). Malignancies were seen in both groups: 1 PSC patient had been diagnosed for cholangiocarcinoma, and 2 PBC patients exhibited hepatocellular carcinoma.

Twenty-three PSC patients (56.1\%) had concomitant IBD (UC, $\mathrm{n}=21$; Crohn's disease, $\mathrm{n}=1$; indeterminate colitis, $\mathrm{n}=1)$. The mean age at diagnosis of IBD (23.2 \pm 15.3 SD years) was significantly lower than the age at diagnosis of PSC ( $31.7 \pm 14.9$ SD years; $p=0.041)$. In 19 of 23 PSC patients with concomitant IBD (82.6\%), the diagnosis of IBD preceded the onset of PSC or was made in the same year. Three PSC patients had a PSC-AIH overlap syndrome. As noted in an earlier study [29], the male predominance in PSC (overall men to women ratio approximately 3:2) was especially pronounced in the subgroup with IBD (78.3\% male). In the subgroup with PSC alone, the sex ratio was more balanced (55.6\% male).

\section{SNPs in the KIAA1109-TENR-IL2-IL21 Gene Cluster}

Are Associated with PSC but Not PBC Susceptibility

The genotype frequencies of all four tested genetic polymorphisms were in accordance with the HardyWeinberg equilibrium. The minor alleles of the four SNPs $r s 13151961(\mathrm{p}=0.013), \mathrm{rs13119723}(\mathrm{p}=0.023)$, rs6822844 ( $\mathrm{p}=0.031)$ and $\mathrm{rs6840978}(\mathrm{p}=0.043)$ were associated with a decreased susceptibility to PSC in our patient cohort (table 2). For the most strongly PSC-associated SNP, rs13151961, the minor allele frequency was 0.061 in PSC patients, whereas it was 0.162 in controls. 
Table 2. Chromosome 4q27 markers in PBC, PSC and healthy controls

\begin{tabular}{|c|c|c|c|c|c|c|c|c|c|c|}
\hline \multirow[t]{2}{*}{ Marker } & \multirow{2}{*}{$\begin{array}{l}\text { Minor } \\
\text { allele }\end{array}$} & \multicolumn{4}{|c|}{$\mathrm{PBC}(\mathrm{n}=124)$} & \multicolumn{4}{|c|}{$\operatorname{PSC}(n=41)$} & \multirow{2}{*}{$\frac{\text { Controls }(\mathrm{n}=1,487)}{\text { MAF }}$} \\
\hline & & MAF & p value ${ }^{a}$ & OR [95 \% CI] & Power & MAF & $\mathrm{p}$ value $\mathrm{a}^{\mathrm{a}}$ & OR [95 \% CI] & Power & \\
\hline rs13151961 & G & 0.154 & 0.857 & $0.94[0.66-1.35]$ & 0.05 & 0.061 & 0.013 & $0.34[0.14-0.83]$ & 0.65 & 0.162 \\
\hline rs13119723 & G & 0.169 & 0.859 & $1.03[0.73-1.45]$ & 0.04 & 0.073 & 0.023 & $0.40[0.17-0.92]$ & 0.58 & 0.166 \\
\hline rs6822844 & $\mathrm{T}$ & 0.150 & 0.718 & $0.92[0.64-1.32]$ & 0.06 & 0.073 & 0.031 & $0.41[0.18-0.95]$ & 0.55 & 0.161 \\
\hline rs6840978 & $\mathrm{T}$ & 0.195 & 0.800 & $1.04[0.75-1.45]$ & 0.04 & 0.098 & 0.043 & $0.46[0.22-0.97]$ & 0.53 & 0.189 \\
\hline
\end{tabular}

Minor allele frequencies (MAF), allelic test p values, odds ratios (OR, shown for the minor allele) with 95\% CIs and power of odds ratio 95\% CI. Significant associations are highlighted in bold.

a Two-tailed p values were calculated by Fisher's exact test analysis of allele counts.

Table 3. Chromosome 4q27 haplotypes in PBC, PSC and healthy controls

\begin{tabular}{|c|c|c|c|c|c|c|c|c|c|c|}
\hline \multirow{2}{*}{$\begin{array}{l}\text { Haplotype } \\
\text { block }\end{array}$} & \multirow{2}{*}{$\begin{array}{l}\text { Allelic } \\
\text { order }\end{array}$} & \multicolumn{4}{|c|}{$\mathrm{PBC}(\mathrm{n}=124)$} & \multicolumn{4}{|c|}{$\operatorname{PSC}(\mathrm{n}=41)$} & \multirow{2}{*}{$\frac{\text { Controls }}{(\mathrm{n}=1,487)}$} \\
\hline & & $\mathrm{HF}$ & $\mathrm{p}$ value $\mathrm{e}^{\mathrm{a}}$ & OR $[95 \% \mathrm{CI}]$ & $\mathrm{P}_{100,000^{\mathrm{b}}}$ & $\mathrm{HF}$ & $\mathrm{p}$ value $\mathrm{a}^{\mathrm{a}}$ & OR $[95 \% \mathrm{CI}]$ & $P_{100,000^{b}}$ & \\
\hline \multirow[t]{3}{*}{1234} & AAGC & 0.791 & 0.66 & $0.93[0.68-1.29]$ & 0.96 & 0.878 & $0.089(0.092)$ & $1.77[0.91-3.46]$ & 0.28 & 0.802 \\
\hline & GGTT & 0.148 & 0.77 & $0.95[0.66-1.37]$ & 0.97 & 0.049 & $\mathbf{0 . 0 0 8 4}(0.0048)$ & $0.28[0.10-0.77]$ & 0.035 & 0.155 \\
\hline & AAGT & 0.045 & 0.17 & $1.56[0.82-2.96]$ & 0.39 & 0.037 & $0.70(0.73)$ & $1.25[0.39-4.04]$ & 1 & 0.029 \\
\hline \multirow{4}{*}{$\begin{array}{l}12 \\
(\text { KIAA1109) } \\
23 \\
(\text { IL2) }\end{array}$} & $\mathrm{AA}$ & 0.836 & 0.99 & $1.00[0.70-1.42]$ & 1 & 0.927 & $\mathbf{0 . 0 2 7}(0.023)$ & $2.49[1.08-5.75]$ & 0.054 & 0.836 \\
\hline & GG & 0.148 & 0.57 & $0.90[0.62-1.30]$ & 0.84 & 0.061 & $\mathbf{0 . 0 1 4}(0.013)$ & $0.34[0.14-0.84]$ & 0.042 & 0.161 \\
\hline & $\mathrm{AG}$ & 0.833 & 0.94 & $1.02[0.72-1.44]$ & 0.99 & 0.915 & $\mathbf{0 . 0 4 6}(0.050)$ & $2.18[1.00-4.75]$ & 0.071 & 0.831 \\
\hline & $\mathrm{GT}$ & 0.150 & 0.79 & $0.95[0.66-1.37]$ & 0.94 & 0.061 & $\mathbf{0 . 0 1 8}(0.013)$ & $0.35[0.14-0.87]$ & 0.045 & 0.157 \\
\hline \multirow{3}{*}{$\begin{array}{l}34 \\
\text { (IL21) }\end{array}$} & GC & 0.805 & 0.88 & $0.98[0.70-1.35]$ & 1 & 0.890 & $0.063(0.064)$ & $1.92[0.95-3.86]$ & 0.23 & 0.809 \\
\hline & TT & 0.150 & 0.72 & $0.94[0.64-1.35]$ & 1 & 0.061 & $\mathbf{0 . 0 1 6}(0.013)$ & $0.34[0.14-0.85]$ & 0.040 & 0.159 \\
\hline & GT & 0.045 & 0.22 & $1.49[0.79-2.83]$ & 0.40 & 0.037 & $0.75(0.74)$ & $1.20[0.37-3.87]$ & 1 & 0.031 \\
\hline
\end{tabular}

Haplotype frequencies (HF), p values, odds ratios (OR) with 95\% CIs and power of odds ratio 95\% CI. $1=$ rs 13151961; $2=$ rs 13119723 ; $3=$ rs6822844; 4 = rs6840978. Significant associations are highlighted in bold and suggestive ones in italics. For each block, all haplotypes with an overall frequency $>1 \%$ were examined. ${ }^{a}$ Individual two-tailed p values from $\chi^{2}$ test analysis of haplotype counts (for PSC, Fisher's exact test $\mathrm{p}$ values in brackets). ${ }^{\mathrm{b}}$ Permutated $\mathrm{p}$ values $(100,000$ permutations).

The odds ratios for the minor allele ranged from 0.34 for rs13151961 to 0.46 for rs6840978. The demonstrated odds ratios of $<1.0$ suggest protective effects of the minor alleles on PSC susceptibility similar to the association observed for other immune-mediated diseases $[8-12,14,16$, $17,20]$. In line with that, homozygosity for the major allele was positively associated with development of PSC when examining rs13151961 ( $\mathrm{p}=0.014$, OR 3.03, 95\% CI $1.18-7.76)$, rs13119723 ( $\mathrm{p}=0.036$, OR $2.53,95 \%$ CI $1.06-$ 6.06 ) or rs6822844 ( $\mathrm{p}=0.037$, OR $2.45,95 \%$ CI 1.02-5.86).

In contrast, we did not find any significant association with PBC concerning allele or genotype frequencies (see table 2 for allele frequencies).

\section{Haplotypes Formed by the SNPs within the}

KIAA1109-TENR-IL2-IL21 Linkage Disequilibrium

Block Are Associated with Susceptibility to PSC

The four SNPs in the KIAA1109-TENR-IL2-IL21 gene cluster are in accentuated linkage disequilibrium to each other (fig. 1). The aforementioned association of the single markers with PSC prompted us to evaluate, whether single haplotypes formed by the SNPs are also associated with susceptibility to PSC or PBC. First, the four SNPs were configured in a single haplotype block. Three haplotypes exhibiting an overall frequency $>0.01$ were observed (table 3). As expected, a haplotype consisting of the four minor alleles was significantly associated with a 
decreased susceptibility to PSC $(\mathrm{p}=0.0084$, OR $0.28,95 \%$ CI 0.10-0.77). A similar haplotype including these four alleles has also a protective effect on UC [10].

In order to better represent the different candidate genes within the chromosome 4q27 region, we analyzed three further haplotypes. One encompassed rs13151961 and rs13119723 within KIAA1109, the two SNPs with the strongest linkage disequilibrium. One included rs13119723 and rs6822844, which distantly surround IL2, and the third one comprised rs6822844 and rs6840978 next to IL21 (fig. 1). All haplotypes formed by the corresponding minor alleles were observed with a significantly lower frequency in PSC patients compared to the healthy controls (table 3). In case of the IL2 and KIAA1109 haplotypes, the assembled major alleles showed a significant positive association with PSC vice versa.

In contrast, we did not detect PBC-associated haplotypes (table 3).

\section{KIAA1109-TENR-IL2-IL21 Variants Are Associated}

with PSC Independently of Concomitant IBD

The herein studied genetic variants are associated with UC, and there is also some evidence for an association with Crohn's disease [8-11]. PSC-IBD is considered a distinct IBD phenotype on its own [3]. The colitis associated with PSC is usually mild and is characterized by a more severe right-sided disease, rectal sparing and backwash ileitis [30]. However, since 23 of our 41 PSC patients (56.1\%) had concomitant IBD, we aimed to investigate whether the observed association with PSC is independent of a co-affection with IBD. Therefore, the haplotypes depicted in table 3 and all four single markers were tested for association with the 18 PSC cases without IBD. Despite the limited sample size, the haplotype containing the four major alleles of rs13151961, rs13119723, rs6822844 and rs6840978 showed significant association with PSC $\left(P_{\mathrm{BL}}=0.033\right.$, OR 4.18, 95\% CI 1.12-24.51). Of the further haplotypes, the one encompassing rs6822844 and rs6840978 next to IL21 was associated with PSC without IBD when combining the two major alleles $\left(P_{\mathrm{BL}}=0.033\right.$, OR 4.02, 95\%CI $1.08-$ 23.53).

With regard to the single markers, rs6840978 did not lose significance when excluding the patients with accompanying IBD $\left(P_{\mathrm{BL}}=0.034\right.$, OR $0.25,95 \%$ CI $0.04-$ 0.94; OR shown for the minor allele). Homozygosity for the major allele of rs6840978 was a risk factor for PSC without IBD $\left(P_{\mathrm{BL}}=0.045\right.$, OR 4.11, $95 \%$ CI $1.05-$ 25.13).

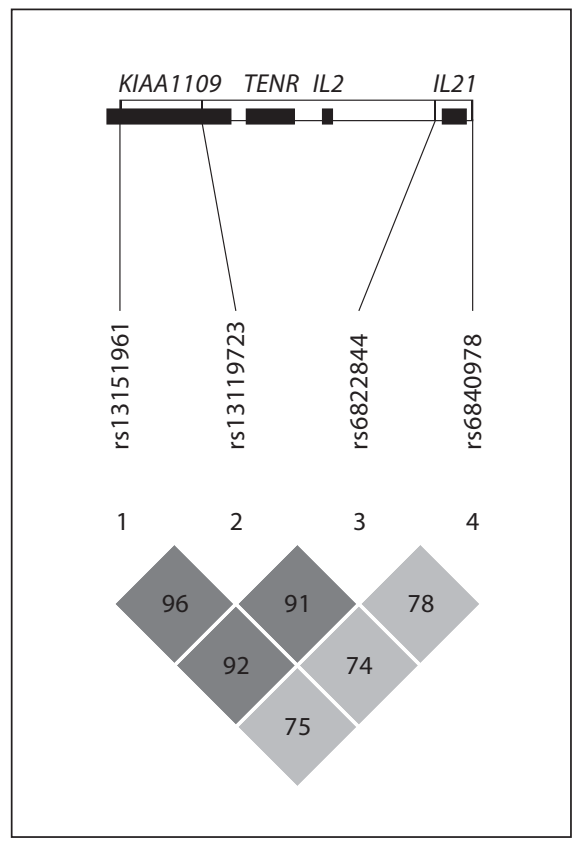

Fig. 1. Overview over the chromosome $4 \mathrm{q} 27$ region. The $439-\mathrm{kb}$ section between rs13151961 and rs6840978 is shown, with genes and LD statistics $\left(\mathrm{r}^{2}\right)$ calculated from the combined dataset of controls, PBC, and PSC patients using Haploview 4.2.

\section{Discussion}

Here, we demonstrate for the first time that the established UC risk locus on chromosome 4q27 [8-11] is associated with susceptibility to PSC.

The contribution of genetic factors to the pathogenesis of PSC is highlighted by a nearly 100 -fold higher prevalence of this disorder in first-degree relatives of PSC patients compared with the general population [31]. According to a recent Swedish study [32], the hazard ratio for UC among first-degree relatives of PSC patients is also raised. Even relatives of PSC patients without IBD have a more than seven times increased risk of developing UC, which strongly suggests the existence of shared genetic susceptibility genes for PSC and UC [32].

However, to date, only two other loci have been described which confer susceptibility to both of these epidemiologically tightly linked diseases. Of 15 known UC loci tested so far, only GPBAR1 and MST1 showed association with overall PSC susceptibility even in a group where the proportion of concomitant IBD was $81 \%$ [7]. Of these two, only one (GPBAR1) was further associated with PSC without IBD in the corresponding subgroup of 77 PSC patients without IBD [7]. 
Interestingly, the same polymorphisms in the KIAA1109-TENR-IL2-IL21 linkage disequilibrium block investigated are also associated with a variety of other immune-mediated diseases. Especially rs6822844 has been extensively studied. It has been associated with celiac disease [12], UC [8-11], rheumatoid arthritis [14, 16], type 1 diabetes [14], psoriatic arthritis [18], juvenile idiopathic arthritis $[19,20]$, systemic lupus erythematosus [17] and Sjögren's syndrome [17].

In all of these diseases, like in PSC, the minor allele frequency of rs6822844 is significantly decreased in cases suggesting protective effects of the minor allele on autoimmune disease susceptibility. On the other hand, the 'wild-type' major allele is a risk factor for the development of autoimmunity.

Insulin-dependent diabetes 3 (IDD3), the corresponding locus in mice, also harbors the four genes KIAA1109, TENR, IL2 and IL21. It is the strongest non-MHC linked susceptibility locus for type 1 diabetes in the nonobese diabetic (NOD) mouse. Several detailed studies have addressed the functional impact of disease-associated alleles within this autoimmunity locus using the NOD mouse model [33-35]. Naturally occurring susceptibility alleles correlate with an approximately twofold reduction in IL-2 expression and hence impair the function of $\mathrm{CD} 4+\mathrm{CD} 25+$ regulatory $\mathrm{T}$ cells, which are critical for maintaining immune homoeostasis [34]. In contrast, protective IDD3 allelic variants promote the expansion and suppressive functions of CD4+Foxp3+ regulatory $\mathrm{T}$ cells and restrain the proliferation and IL-17 production of pro-inflammatory CD4+ T cells by boosting IL- 2 expression [33]. IL-2 is essential for TGF- $\beta$ to convert naive CD4+CD25- cells to CD25+Foxp3+ regulatory $\mathrm{T}$ cells [36] and via STAT5 constrains the generation of Th17 cells [37], which are implicated in the pathogenesis of autoimmune diseases.

Mice with a disrupted IL2 gene develop an UC-like disease [38]. Defective IL-2 production has also been described for freshly isolated liver derived $\mathrm{T}$ lymphocytes from PSC patients. Compared with patients with PBC, $\mathrm{AIH}$, or normal controls, significantly higher levels of tumor necrosis factor (TNF)- $\alpha$ and IL-1 $\beta$ but lower levels of IL-2, IL-10, and interferon- $\gamma$ were found in the supernatants of mitogen-stimulated lymphocyte cultures [39]. Like IL-2 [40], IL-10 is considered an anti-inflammatory cytokine promoting immune tolerance [41].

Another study on the role of autoimmunity-related alleles in IDD3, the mouse homologue for human chromosome $4 \mathrm{q} 27$, indicates that a loss of locus parity between IL2 and IL21 decreases IL-2 mRNA stability and ensures high IL-21 production as a basis for autoimmunity [35]. IL-21 plays a key role in Th17 differentiation [42], leads to a down-regulation of FOXP3 expression in CD4+ T cells [43] and renders CD4+CD25- T cells resistant to regulatory T-cell-mediated suppression [44]. The opposing functions of IL-2 and IL-21 in tolerance and immunity underpin the disastrous effect of a dysregulated expression of these two cytokines.

Unlike in PSC, we could not detect any impact of the KIAA1109-TENR-IL2-IL21 SNPs on susceptibility to $\mathrm{PBC}$. This finding is in line with a recent genome-wide association study comprising these SNPs [6]. Taking into account that for rs13151961, our study would have had a $98 \%$ power to reveal a difference in minor allele frequency, like it was observed in PSC, it is unlikely that genetic variation in the IL2/IL21 region plays a major role in the pathogenesis of $\mathrm{PBC}$. However, a minor effect on PBC susceptibility cannot be excluded, since the power of our study to detect a lower difference in minor allele frequency of 5\%, for instance, for rs 13151961 , was only $51 \%$.

The aforementioned recent genome-wide analysis in PBC [6] could find an association with rs6838639 on chromosome $4 \mathrm{q} 27$ about $200 \mathrm{~kb}$ away from the KIAA1109-TENR-IL2-IL21 linkage disequilibrium block in its discovery panel. This finding, however, could not be verified in two replication cohorts [6].

In contrast to IL2 and IL21, KIAA1109 is a predicted gene of largely unknown function [12]. TENR (testis nuclear RNA-binding) expression is restricted to testes making it an unlikely disease gene for PSC [12].

In conclusion, we here provide first evidence that the KIAA1109-TENR-IL2-IL21 linkage disequilibrium block represents a shared susceptibility locus for UC and PSC. IL2 and IL21 are the likely disease genes. Further replication studies in larger cohorts are required to confirm this disease locus.

\section{Acknowledgments}

This work is part of the unpublished degree thesis of J.S. G.U.D. is recipient of a research grant from the Else KrönerFresenius-Stiftung (P65/08//A56/08). S.B. was supported by grants from the DFG (BR 1912/5-1), by the Ludwig-Demling Grant 2007 from DCCV e.V., and by grants of Ludwig-Maximilians University Munich (Excellence Initiative, Investment Funds 2008 and FöFoLe program). None of the authors and co-authors has competing interests related to the manuscript. 


\section{References}

1 Chapman R, Cullen S: Etiopathogenesis of primary sclerosing cholangitis. World J Gastroenterol 2008;14:3350-3359.

2 Gershwin ME, Mackay IR: The causes of primary biliary cirrhosis: convenient and inconvenient truths. Hepatology 2008;47:737745 .

3 Loftus EV Jr, Harewood GC, Loftus CG, et al: PSC-IBD: a unique form of inflammatory bowel disease associated with primary sclerosing cholangitis. Gut 2005;54:91-96.

4 Loftus EV, Sandborn WJ, Lindor KD, et al: Interactions between chronic liver disease and inflammatory bowel disease. Inflamm Bowel Dis 1997;3:288-302.

5 Zhernakova A, van Diemen CC, Wijmenga C: Detecting shared pathogenesis from the shared genetics of immune-related diseases. Nat Rev Genet 2009;10:43-55.

6 Hirschfield GM, Liu X, Xu C, et al: Primary biliary cirrhosis associated with HLA, IL12A, and IL12RB2 variants. N Engl J Med 2009;360:2544-2555

7 Karlsen TH, Franke A, Melum E, et al: Genome-wide association analysis in primary sclerosing cholangitis. Gastroenterology 2010;138:1102-1111.

8 Festen EA, Goyette P, Scott R, et al: Genetic variants in the region harbouring IL2/IL21 associated with ulcerative colitis. Gut 2009; 58:799-804.

9 Glas J, Brand S: Consolidation of evidence for association of the KIAA1109-TENR-IL2IL21 rs6822844 variant with Crohn's disease response. Am J Gastroenterol 2010;105: 1206-1207.

10 Glas J, Stallhofer J, Ripke S, et al: Novel genetic risk markers for ulcerative colitis in the IL2/IL21 region are in epistasis with IL23R and suggest a common genetic background for ulcerative colitis and celiac disease. Am J Gastroenterol 2009;104:1737-1744.

11 Marquez A, Orozco G, Martinez A, et al: Novel association of the interleukin 2-interleukin 21 region with inflammatory bowel disease. Am J Gastroenterol 2009;104:19681975.

12 van Heel DA, Franke L, Hunt KA, et al: A genome-wide association study for celiac disease identifies risk variants in the region harboring IL2 and IL21. Nat Genet 2007;39: 827-829.

13 Barrett JC, Clayton DG, Concannon P, et al: Genome-wide association study and metaanalysis find that over 40 loci affect risk of type 1 diabetes. Nat Genet 2009;41:703-707.

14 Zhernakova A, Alizadeh BZ, Bevova M, et al: Novel association in chromosome 4q27 region with rheumatoid arthritis and confirmation of type 1 diabetes point to a general risk locus for autoimmune diseases. Am J Hum Genet 2007;81:1284-1288.
15 Todd JA, Walker NM, Cooper JD, et al: Robust associations of four new chromosome regions from genome-wide analyses of type 1 diabetes, Nat Genet 2007;39:857-864.

16 Daha NA, Kurreeman FA, Marques RB, et al: Confirmation of STAT4, IL2/IL21, and CTLA4 polymorphisms in rheumatoid arthritis. Arthritis Rheum 2009;60:12551260.

17 Maiti AK, Kim-Howard X, Viswanathan P, et al: Confirmation of an association between rs6822844 at the IL2-IL21 region and multiple autoimmune diseases: evidence of a general susceptibility locus. Arthritis Rheum 2010;62:323-329.

18 Liu Y, Helms C, Liao W, et al: A genome-wide association study of psoriasis and psoriatic arthritis identifies new disease loci. PLoS Genet 2008;4:e1000041.

19 Albers HM, Kurreeman FA, Stoeken-Rijsbergen G, et al: Association of the autoimmunity locus 4q27 with juvenile idiopathic arthritis. Arthritis Rheum 2009;60:901-904.

20 Hinks A, Eyre S, Ke X, et al: Association of the AFF3 gene and IL2/IL21 gene region with juvenile idiopathic arthritis. Genes Immun 2010;11:194-198.

21 Sawalha AH, Kaufman KM, Kelly JA, et al Genetic association of interleukin-21 polymorphisms with systemic lupus erythematosus. Ann Rheum Dis 2008;67:458-461.

22 Saarinen S, Olerup O, Broome U: Increased frequency of autoimmune diseases in patients with primary sclerosing cholangitis. Am J Gastroenterol 2000;95:3195-3199.

23 Gershwin ME, Selmi C, Worman HJ, et al: Risk factors and comorbidities in primary biliary cirrhosis: a controlled interviewbased study of 1032 patients. Hepatology 2005;42:1194-1202.

24 Kaplan MM, Gershwin ME: Primary biliary cirrhosis. N Engl J Med 2005;353:1261-1273.

25 Weismuller TJ, Wedemeyer J, Kubicka S, et al: The challenges in primary sclerosing cholangitis - aetiopathogenesis, autoimmunity, management and malignancy. J Hepatol 2008;48(suppl 1):S38-S57.

26 Barrett JC, Fry B, Maller J, et al: Haploview: analysis and visualization of LD and haplotype maps. Bioinformatics 2005;21:263-265.

27 Fay MP: Confidence intervals that match Fisher's exact or Blaker's exact tests. Biostatistics 2010;11:373-374.

28 Chen B, Wilkening S, Drechsel M, et al SNP_tools: a compact tool package for analysis and conversion of genotype data for MSExcel. BMC Res Notes 2009;2:214.

29 Henckaerts L, Fevery J, Van Steenbergen W, et al: CC-type chemokine receptor 5-Delta32 mutation protects against primary sclerosing cholangitis. Inflamm Bowel Dis 2006; 12 : 272-277.
30 Saich R, Chapman R: Primary sclerosing cholangitis, autoimmune hepatitis and overlap syndromes in inflammatory bowel disease. World J Gastroenterol 2008;14:331-337.

31 Bergquist A, Lindberg G, Saarinen S, et al: Increased prevalence of primary sclerosing cholangitis among first-degree relatives. J Hepatol 2005;42:252-256.

32 Bergquist A, Montgomery SM, Bahmanyar $S$, et al: Increased risk of primary sclerosing cholangitis and ulcerative colitis in first-degree relatives of patients with primary sclerosing cholangitis. Clin Gastroenterol Hepatol 2008;6:939-943.

33 Sgouroudis E, Albanese A, Piccirillo CA: Impact of protective IL-2 allelic variants on $\mathrm{CD} 4+$ Foxp3+ regulatory $\mathrm{T}$ cell function in situ and resistance to autoimmune diabetes in NOD mice. J Immunol 2008;181:6283-6292.

34 Yamanouchi J, Rainbow D, Serra P, et al: Interleukin-2 gene variation impairs regulatory $\mathrm{T}$ cell function and causes autoimmunity. Nat Genet 2007;39:329-337.

35 McGuire HM, Vogelzang A, Hill N, et al: Loss of parity between IL-2 and IL-21 in the NOD Idd3 locus. Proc Natl Acad Sci USA 2009;106:19438-19443.

36 Zheng SG, Wang J, Wang P, et al: IL-2 is essential for TGF-beta to convert naive CD4+CD25- cells to CD25+Foxp3+ regulatory $\mathrm{T}$ cells and for expansion of these cells. J Immunol 2007;178:2018-2027.

37 Laurence A, Tato CM, Davidson TS, et al: Interleukin-2 signaling via STAT5 constrains T helper 17 cell generation. Immunity 2007; 26:371-381.

38 Sadlack B, Merz H, Schorle H, et al: Ulcerative colitis-like disease in mice with a disrupted interleukin-2 gene. Cell 1993;75:253261

39 Bo X, Broome U, Remberger M, et al: Tumour necrosis factor alpha impairs function of liver derived $\mathrm{T}$ lymphocytes and natural killer cells in patients with primary sclerosing cholangitis. Gut 2001;49:131-141.

40 Malek TR: The biology of interleukin-2. Annu Rev Immunol 2008;26:453-479.

41 Saraiva M, O'Garra A: The regulation of IL10 production by immune cells. Nat Rev Immunol 2010;10:170-181.

42 Yang L, Anderson DE, Baecher-Allan C, et al: IL-21 and TGF-beta are required for differentiation of human $\mathrm{T}(\mathrm{H}) 17$ cells. Nature 2008;454:350-352.

43 Li Y, Yee C: IL-21 mediated Foxp3 suppression leads to enhanced generation of antigen-specific CD8+ cytotoxic T lymphocytes. Blood 2008;111:229-235.

44 Peluso I, Fantini MC, Fina D, et al: IL-21 counteracts the regulatory $\mathrm{T}$ cell-mediated suppression of human CD4+ T lymphocytes. J Immunol 2007;178:732-739. 